\title{
On the Meta-Analysis Review Towards efficient mechanisms for resource, energy, and data management in the fog computing
}

\author{
Professor Dr. Sorush Niknamian \\ Ph.D. in Cell and Molecular Biology, CSci (Chartered Scientist), RSciTech (Registered Science Technician), \\ RSci (Registered Scientist) ID: A-0008833, Science Council, United Kingdom and Indiana Cancer Consortium \\ (ICC) certified. Registered Researcher in the U.S. Government's System for Award Management. Registered \\ Scientist at National Center for Biotechnology Information. Medical Student at Liberty University, United \\ States of America, Military Medicine. BSc in Mechatronics Engineering and BSc in applied Mathematics.
}

\begin{abstract}
Fog computing is an architecture that uses collaborative end-user edge devices to carry out a large amount of storage, transmission, configuration, and module function. In this computing environment, management issue is the process of managing, monitoring and optimizing the correlated components for improving the performance, availability, security and any fundamental operational requirement. The management strategies have a great impact on the fog computing, but, as far as we know, there is not a comprehensive and systematic study in this field. Hence, this paper classifies the management strategies into three main categories, including resource, energy and data management. In addition, it defines the new challenges in each of these categories. Finally, the differences between the reviewed strategies are investigated in terms of scalability, reliability, time, and queries attributes along with providing the main directions for future research.
\end{abstract}

Keywords: Fog computing, Management, Resource, Data, Energy

\section{Introduction}

Nowadays, the data transmission is facilitated through Internet and web services [1]. Fog computing, as a new Internet-based computing paradigm, facilitates wireless data transference to the distributed devices based on computing, storage, and networking services, which are authorized among end systems and data centers [2]. The edge of the network is a place for providing computing services and also it arranges the modern applications and services for the future of the Internet [3]. The components of data-processing or analysis applications are included in the fog computing (Dastjerdi, Gupta, Calheiros, Ghosh, \& Buyya, 2016). Fog computing defines the representative applications and mentions various aspects of issues in designing and implementing computing systems [4]. Nevertheless, some questions are 
provided in order to discuss the Quality of Service (QoS), management, accessibility, security, and privacy in fog computing [5].

On the other hand, it is necessary to have a dynamic and powerful management strategy because of the distributed character of the fog computing [6]. The real-time nature of the system is concerned with efficient management strategy associated with distributed objects/systems and their related devices. The fog management facilitates computing management, networking, and storage services among end devices [7].

Despite the importance of the management strategies in the fog computing, as far as we know, there is not any systematic review about analyzing the importance of management strategies in the fog environments. Searching existing techniques and comparing differences among the fog management strategies are considered as the main goals of this paper. The fog management strategies are classified into three main categories, including resource, energy, and data management. Investigating the management strategies with a specific focus on fog computing is the first step in this survey. Briefly, the main purposes of this paper are as follows:

- Presenting an overview of the current challenges associated with management strategies in the fog computing.

- Presenting a systematic review of the current management strategies in the fog computing.

- Exploring the future challenges and their roles in the management of fog computing.

- Highlighting the important areas of future research to improve the use of management strategies in fog computing.

The rest of this paper is organized as follows: Section 2 discusses the background and related terminology. Section 3 discusses some related works. A systematic literature review is presented in Section 4. A review of selected papers is discussed in section 5. Results and comparisons are mentioned in Section 6. Open issues and conclusion are discussed in sections 7 and 8 , respectively.

\section{Background and related terminologies}

This section describes the background related to fog and cloud computing, their advantages and disadvantages. The key concepts of this study are also discussed. The cloud and fog 
computing will be discussed first. Then, managing mechanisms and a brief overview of existing studies are examined in fog environment.

\subsection{Cloud Computing}

Cloud computing, as a model for allowance of ubiquitous and on-demand network access to a shared pool of configurable resources, can be rapidly provisioned and released with minimal provider interaction, with charges potentially being levied for its use [8]. Thus, delocalization of computing infrastructure is quite simple. Cloud computing is the operation of computing power or storage on remote servers by means of a network (generally the Internet) [9]. The cloud services are divided into three main types, such as [10]:

- IaaS - Infrastructure as a Service;

- PaaS - Platform as a Service;

- SaaS - Software as a Service.

In this environment, latency and intermittent connectivity issues between cloud services and smart devices are also very important. The performance of the application in real time depends on a high-speed Internet connection to the cloud service, and its nearness to users [11].

\subsection{Fog computing}

Cloud computing is a way to process and store the data. However, cloud computing is still unable to solve some challenges such as increasing demands of latency-sensitive applications and limitation of network bandwidth [12]. Fog computing allows delivering the right data at the right time to people on any device. The model for data analytics involves a centralized data warehouse manual data manipulation and investigation, with the vast amount of data on internet of everything pouring. It is difficult to find and act on the right data if the data is housed in centralized data warehouses Naranjo, Shojafar, Vaca-Cardenas, et al. (2016). Although the centralized cloud computing provides many resources for computation-intensive tasks, unpredictable and unstable communication latency between the mobile users and the cloud causes to handle latency-sensitive mobile computing tasks [13]. Therefore, fog computing, as a new computing paradigm, is proposed to complete the cloud solution [14]. Cloud services are extended to the network edge through fog computing, and the computation, communication, and storage become closer to edge devices and end-users to improve latency, network bandwidth, security and privacy [15]. Fog computing emerged in an effort to address the exponential proliferation of connected devices in the IoT world. Therefore, this aspect should be scrutinized and tested thoroughly in future studies. However, the lack of supporting 
infrastructure for fog computing has hindered the effort to evaluate the applications in a larger scale [16].

Fog computing provides means to utilize available resources close to the edge of the network O Skarlat (2017).

Provided services by the cloud are extended to the edge of the network via fog computing [17]. Fog computing includes components of an application running on the cloud and the devices between endpoints and the cloud, i.e. routers and smart gateways [18]. The mobility, resource and interface heterogeneity are supported by fog computing. The analyzed data is distributed in applications that need low latency with a wide and dense geographical distribution [19]. Fog computing takes advantages of both edge and cloud computing as it moves towards the edge if there is a need to approach the endpoints [20]. Also, it penetrates in cloud resources for ondemand scalability [21].

Previous researchers brought the cloud facilities closer to the mobile user by proposing the concept of fog computing. It aims at provisioning resources, like computation power, storage, and business logic, at the edge of the wireless access network [22]. End devices, fog, and cloud are used to form a three-layer hierarchical service delivery model and support a range of applications such as web content delivery, augmented reality, and large data analysis. Many privacy and security issues in the cloud computing can be solved under the influence of fog computing because it is considered as an unimportant format for the cloud.

\subsection{Fog architecture}

Fog architecture contains 3 basic layers, including mobile devices, fog nodes, and cloud infrastructure as shown in Fig. 1 [23]. The mobile device layer contains all types of mobile devices such as mobile phones and Internet of Thing (IoT) devices. However, the resources in fog layer are capable to control network traffic and host many applications such as image processing, smart tracking, and gaming. Moreover, fog layer is responsible for optimization of mobile resources and large data received from mobile devices before sending it to the cloud. Furthermore, fog layer provides cloud services in the close proximity to mobile devices. The cloud layer contains powerful resources that can be used for mining patterns, large data analytics, etc. [24] Finally, the technical support for IoT, Cyber-Physical Systems (CPS) and mobile Internet are provided through this architecture to offer proficient data processing and 
storing services. Monitoring or controlling the entities and objects that exist in the physical world require the combination of computing, communications, and storage by CPS [25].

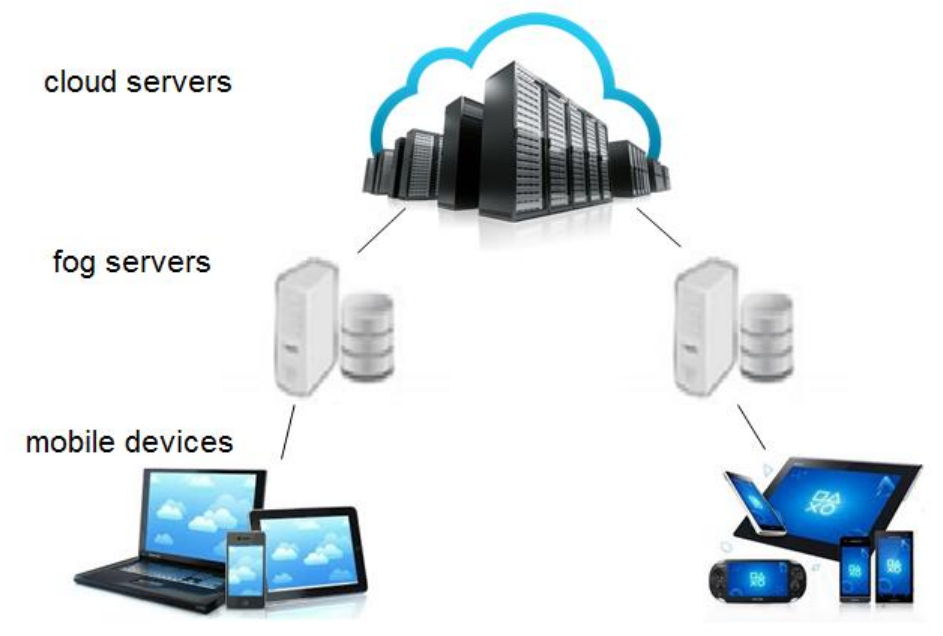

Fig 1. Fog computing architecture (Yi, Qin, \& Li, 2015)

\subsection{Edge computing}

Additional computing and storage resources are located at the edge to handle the localized service requests using local resources and connections. Fog server at different locations needs to adopt its services with management and maintenance costs [26]. Edge computing allows strong technologies perform the computation at the edge of the network. Edge, as any network and computing resources, is placed along the path between data sources and cloud data centers. For example, a gateway is an edge between cloud and home things, and a Micro Data Center (MDC) and a cloudlet is the edge between a mobile device and cloud. The performance of computation at the proximity of data resources is considered as the rationale of edge computing [27].

\subsection{Fog management}

Large data becomes the current critical research focusing on the growth of IoT and IoE technologies. The cloud computing as a current technology is not efficient to address the requirements of large data management. Hence, new technologies are required to decrease the complexity and management efforts and to boost large data processing. A load of large data in cloud computing is reduced by preprocessing raw sensory data [28]. One of the main concerns 
in the era of the Internet of Moving Things (IoMT) is the risk of overflowing a system due to billions of IoMT devices generating a huge volume of data streams that need to be sent out to the cloud for processing and analytic tasks. The recent studies have described the importance of combining edge and cloud computing to address some issues such as speed execution, accuracy, bandwidth cost and privacy. Some important metrics to evaluate the efficiency of fog manager are described as follows:

Time: Runtime or execution time refers to the time when a fog management program is running.

Cost: It includes the cost of running or managing fog at a specified time interval.

Flexibility: The ease of the fog's respond to uncertainty is considered as its flexibility.

Scalability: Scalability refers to the capability of a fog system to handle a growing amount of works and users.

Performance: Performance is the amount of work accomplished by a fog system.

Security: The security protects the hardware, software or information of fog systems, as well as the disruption or misdirection of the services they provide.

Complexity: The computational complexity, or simply complexity of an algorithm is the number of resources required for running it. Here, the computational complexity is the minimum of the complexities of all possible algorithms for the managing problem.

Availability: The probability that a fog system acts at a given time is called availability, i.e. the amount of time that some fog devices actually performs as the percentage of total time required.

Efficiency: The ratio of useful work to fog resources is called efficiency. In other words, it is the ratio of the output to the input of a fog system.

\subsection{Difference between fog and cloud computing}

Cost efficiency, backup and recovery, easy access to information, quick deployment, and automatic software integration are the advantages of cloud computing but there are some disadvantages such as technical issues related with an Internet connection, security issues, limited control and flexibility, dependency, vendor lock-in, and increased vulnerability. Fog computing paradigm could be seen as an extension to cloud computing. It aims to reduce the load by moving workloads, services, applications and huge data near network edge. The main differences between fog and cloud computing are shown in table 1 [29]. 
Table 1. Fog and cloud computing differences

\begin{tabular}{|c|c|c|}
\hline Parameters & Cloud computing & Fog computing \\
\hline Client and server distance & Multiple hops & Single hop \\
\hline Latency & High & Low \\
\hline Security & Less secure & More secure \\
\hline Vulnerability & High & Low \\
\hline Geographical distribution & Centralized & Distributed \\
\hline Mobility & Limited support & Full support \\
\hline Number of server nodes & Few & Large \\
\hline
\end{tabular}

\section{Materials and Methods}

There are many types of research in the field of fog computing and related strategies. However, the management strategies in the fog computing have attracted less attention for establishing the review research. This section presents some survey papers that are proposed in the related fields to highlight the necessity for doing this paper.

Yi, Hao, Qin, and Li (2015) have presented one of the significant surveys of the fog computing. The description of fog computing and related concepts are considered in this survey. Also, it presents the representative application scenarios and recognizes different aspects of issues related to the designing and executing of fog systems. This survey has a specific focus on fog computing but it does not consider the fog management. [30]

One of the significant surveys of the energy efficient techniques in fog computing presented by Amendola, Cordeschi, and Baccarelli (2016). The main lesson stemming from the results reported in this paper is that a key challenge for coping with the unpredictable large volume of data generated by IoE-based applications is the design of a spectrum of hierarchically organized networked computing nodes, namely, proximate Fog and remote Cloud data centers. The final goal is the adaptive energy-efficient reconfiguration and orchestration of the virtualized computing-plus-communication resources available at the computing nodes and thing devices under real-time constraints on the allowed computing-plus-communication delay and service latency. In order to attain this goal, the design of distributed and adaptive resource orchestrators 
that jointly perform the energy and delay-efficient allocation and scheduling of the offered workload over the full spatial spectrum of the available computing nodes is a still challenging research issue. [31]

Furthermore, Manvi and Shyam (2014) have presented a survey of resource management strategies in the cloud computing. Some schemes such as resource provisioning, allocation, topography, and conformity are considered in this paper. Many problems, including flexibility, customization, scalability, and reusability are also mentioned in this paper to manage the cloud resources. They finally have emphasized that some performance metrics such as bandwidth, delay, reliability, and security should be considered in designing an efficient resource management scheme. However, the paper only discusses the management strategies in the cloud. [32]

M Shojafar (2016)have evaluated a cognitive computing-inspired scheduler for the joint adaptive tuning of the: (i) input traffic; (ii) output traffic; and, (iii) resource reconfiguration and consolidation, of virtualized Fog platforms that support vehicular TCP/IP connections. The overall goal is the energy-saving support of QoS-demanding computing intensive delaysensitive I2V services. Remarkable features of the developed joint scheduler are that: (i) its implementation is distributed and adaptive; (ii) it minimizes the energy consumed by the overall NetFC platform for computing, intra-Fog communication and wireless transmission over the vehicular TCP/IP connection; and, (iii) despite the unpredictable time-varying nature of the states of underlying TCP/IP vehicular connections, it is capable to provide hard QoS guarantees, in terms of minimum per-connection instantaneous wireless transmission rate, maximum instantaneous rate-jitter and maximum queuing-plus-computing delay. Actual performance of the proposed scheduler has been numerically tested under both synthetic and real-world input traffic, various mobility conditions and settings of the networked Fog platform. This work can be extended in some directions of potential interest. Just as an example, closed networked multi-tier computing infrastructures may be considered for the support of delay-tolerant session-based services [33]. Since, in this application scenario, traffic arrivals could be allowed, live migration of VMs could be also forecast for attaining additional energy savings.

Also, Jennings and Stadler (2015) have reviewed the recent literature and publications about cloud resource management and highlighted key results. A framework is outlined for cloud resource management to assemble the state-of-the-art analysis. Five challenges are also 
considered for future research related to providing predictable performance for cloud-hosted applications and accepting global manageability for the cloud based on their examination. They only have included the main analysis and challenges in the cloud management and the management parameters with a special focus on the fog is not considered. [34]

Briefly, a pure systematic and comprehensive literature review of the current management strategies in the fog computing is not presented through these surveys. The future challenges and the crucial role of management strategies are discussed in these techniques. However, none of them provides a systematic and comprehensive literature review about analyzing the importance of new issues and techniques in these fields. So, three questions are formalized in the next part in order to select the most important fog management strategies for reviewing. The answering of each question will highlight the importance of these strategies, current challenges, and future trends.

\section{Systematic literature review}

There is a strong focus on management strategies to advance their concepts in the fog computing system. Three research questions are developed to address the key concerns of management strategies in fog computing. In the following subsection, these questions are formalized.

\subsection{Question formalization}

This section aims to outline the most relevant issues and challenges in the fog management by addressing the following research questions:

RQ1: How much running management methods meet the main fog management metrics?

This question aims to assess the current management approaches based on the primary managing metrics in the fog environment.

RQ2: What is the importance of management strategies in the fog environment?

The importance, efficacy, and role of management strategies in the fog computing are defined through this question.

RQ3: Which issues are specified with regard to fog management in the future?

This question aims to identify the role of management strategies, the challenges, and techniques used to manage resource, data, and energy in the fog computing. 
A review protocol is formulated after identifying the need for research. There are different processes to develop the following plans such as search query, source and criteria selection, quality evaluation criteria and data extraction.

\subsection{Article selection process}

A journal and conference papers for the search query are chosen through Google Scholar. The publishers are classified and analyzed in order to summary relevant results. The word "allintitle" in the Scholar.Google.com is used to find the articles with the title of fog, management, and computing. Also, some of the most reliable databases such as IEEE, ACM, and Elsevier are utilized for attending the search process.

\subsubsection{Search method}

Defining keywords are helpful in developing search strings for academic databases. Search strings are defined by recognizing alternative spellings for each of the question components and associate them by utilizing the Boolean OR and Boolean AND [35]. So, some keywords such as "fog computing", "cloud" and "management" are selected. The questions are defined using the results of the initial analysis as a plan and passing some steps to study the coverage of the results. The search string is applied to abstract, titles, and the main body of the articles as far as possible to develop the scope.

\subsubsection{Selection of sources}

Google scholar is adapted to our data source because it covers all available articles. Also, the word "allintitle" is used to find the articles with the title of fog, management, and computing. As a result, the search process covers the available articles that are scientifically confirmed such as IEEE, ACM, Taylor, Wiley, Springer, and Elsevier.

\subsubsection{Selection criteria}

The first stage includes the automated search based on the keywords. Consequently, 42 articles are selected from journals, conference papers, books, chapters, and notes. Some criteria should be specified to select the relevant articles. It is necessary to include or exclude the irrelevant papers and just use the mentioned keywords. The irrelevant articles, books, and surveys are recognized and removed after using a filter. Then, 30 articles are obtained and classified for more investigation. 11 articles are selected based on full text, data extraction, and quality assessment to do a more detailed examination. Also, their advantages and disadvantages are highlighted. The process of article selection is shown in Table 3. 
Table 2. Summary of the inclusion/exclusion criteria for articles selection

\begin{tabular}{|c|c|c|}
\hline Inclusion/ Exclusion & Criterion & Rational \\
\hline Inclusion 1 & $\begin{array}{l}\text { A study that clearly mentioned the } \\
\text { managing strategies in the fog } \\
\text { computing. }\end{array}$ & $\begin{array}{l}\text { We need articles that directly proposed } \\
\text { management strategy in the fog } \\
\text { computing. }\end{array}$ \\
\hline Inclusion 2 & $\begin{array}{l}\text { A study that is developed by academics } \\
\text { or practitioners. }\end{array}$ & $\begin{array}{l}\text { Both academic and industrial solutions are } \\
\text { relevant to this study. }\end{array}$ \\
\hline Inclusion 3 & $\begin{array}{l}\text { A study that is published in the fog } \\
\text { computing field. }\end{array}$ & $\begin{array}{c}\text { Management in the fog is the reference } \\
\text { field. }\end{array}$ \\
\hline Inclusion 4 & Articles that are written in English. & $\begin{array}{l}\text { The papers are written in other languages } \\
\text { than English are removed. }\end{array}$ \\
\hline Exclusion & $\begin{array}{l}\text { All studies except journal and } \\
\text { conference papers are excluded. }\end{array}$ & $\begin{array}{l}\text { Masters dissertations, Ph.D. thesis, } \\
\text { textbooks and unpublished working papers } \\
\text { are omitted. }\end{array}$ \\
\hline
\end{tabular}

Table 3. Stages of articles selection

\begin{tabular}{cc}
\hline Stage & Process \\
\hline Stage 1 & Automated search by keywords \\
Stage 2 & Applying criteria \\
Stage 3 & Selection based on full text \\
\hline
\end{tabular}

The number of published journal articles between 2010 and 2017 are shown in Fig. 2. The obtained 75 articles are shown in Figure 3 where $55 \%$ of the articles are related to IEEE, 10\% are related to Elsevier, $6 \%$ are related to Taylor, 3\% are related to Springer, and 3\% are related to ACM journal articles. 


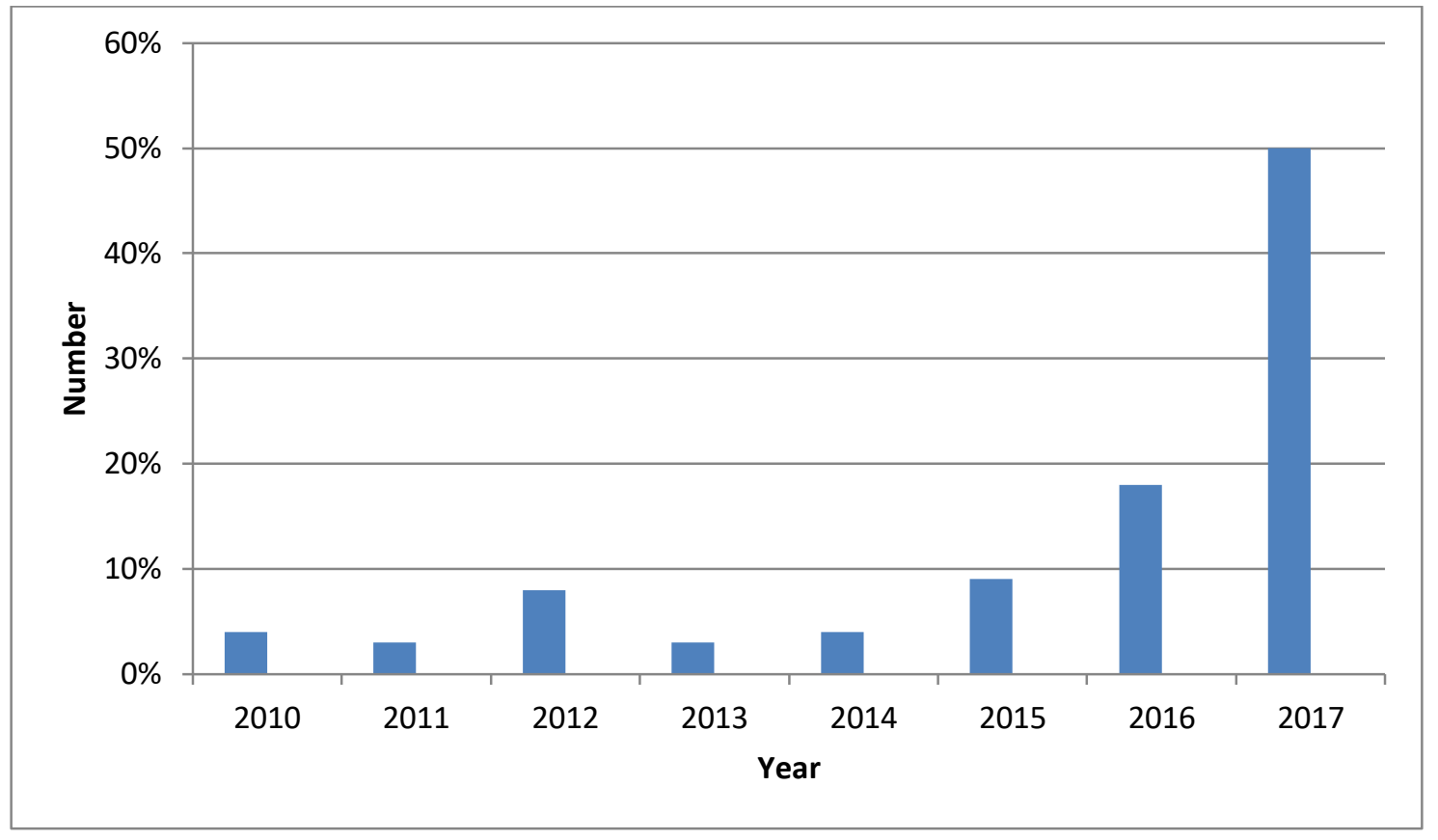

Fig 2. Distribution of articles by year of publication



Publisher

Fig 3. Percentage of published articles b any publisher 

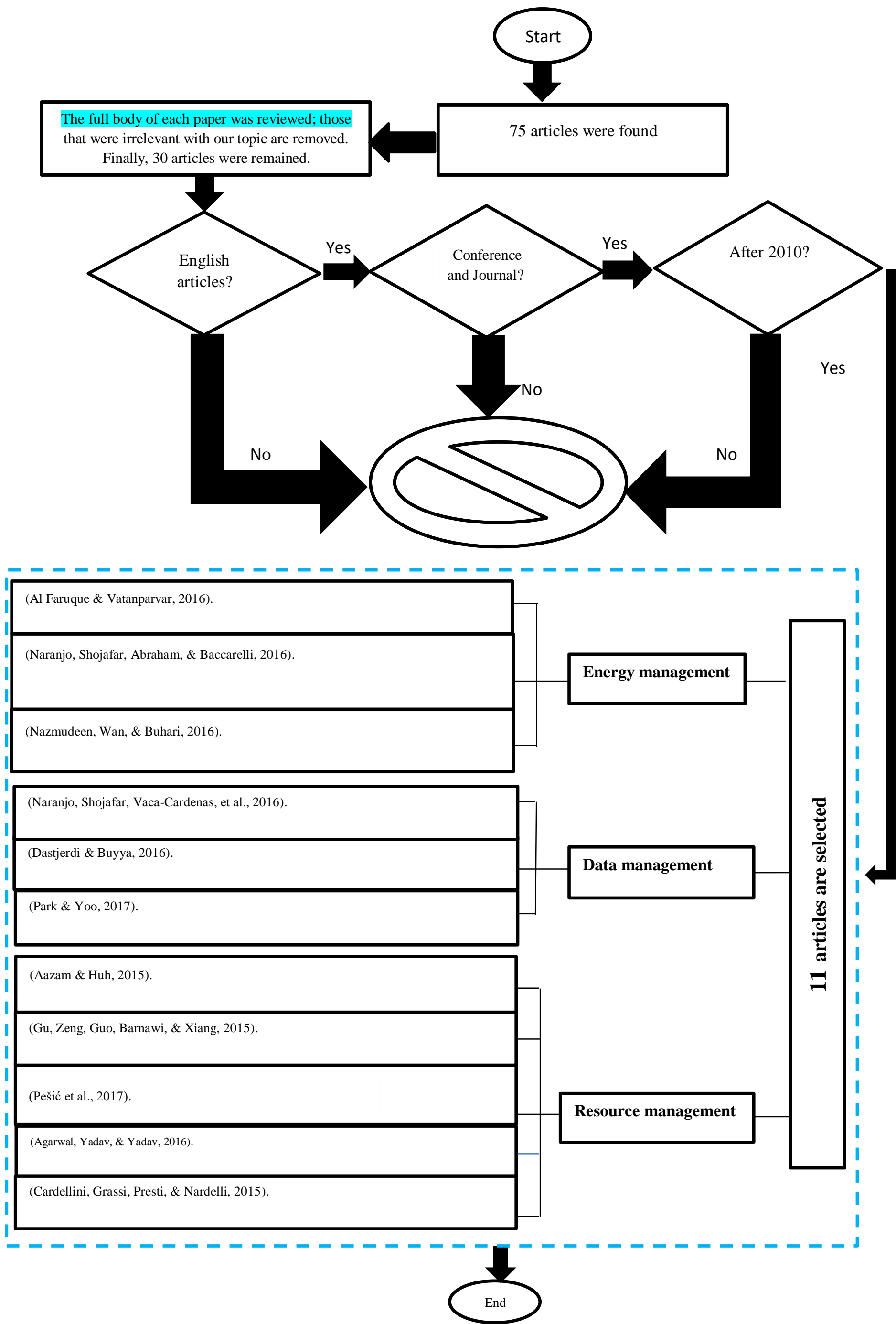
Table 4. Details of the selected articles

\begin{tabular}{|c|c|c|c|}
\hline Publisher & Journal and Conference Name & Author \& Year & Selected? \\
\hline \multirow{16}{*}{ IEEE } & IEEE Power and Energy Magazine & $\begin{array}{c}\text { (Panciatici, Bareux, \& } \\
\text { Wehenkel, 2012) }\end{array}$ & $\mathrm{NO}$ \\
\hline & Information Reuse and Integration & (Dsouza et al., 2014) & $\mathrm{NO}$ \\
\hline & $\begin{array}{l}\text { Sensing, Communication, and Networking- } \\
\text { Workshops (Cirani, Ferrari, Iotti, \& Picone) }\end{array}$ & (Cirani et al., 2015) & $\mathrm{NO}$ \\
\hline & $\begin{array}{c}\text { Sensing, Communication, and Networking- } \\
\text { Workshops } \\
\text { (Cirani et al.) } \\
\end{array}$ & $\begin{array}{l}\text { (Cardellini, Grassi, Lo } \\
\text { Presti, \& Nardelli, } \\
\text { 2016) } \\
\end{array}$ & YES \\
\hline & $\begin{array}{c}\text { Advanced Information Networking and } \\
\text { Applications (Aazam \& Huh) } \\
\end{array}$ & (Aazam \& Huh, 2015) & $\overline{\text { YES }}$ \\
\hline & $\begin{array}{c}\text { Transactions on Emerging Topics in } \\
\text { Computing }\end{array}$ & (Gu et al., 2015) & YES \\
\hline & $\begin{array}{l}\text { Hot Topics in Web Systems and } \\
\text { Technologies (HotWeb) }\end{array}$ & (Yi, Li, et al., 2015) & NO \\
\hline & $\begin{array}{l}\text { Cloud Networking } \\
\text { (Cloudnet) }\end{array}$ & $\begin{array}{c}\text { (Amendola et al., } \\
\text { 2016) } \\
\end{array}$ & NO \\
\hline & $\begin{array}{l}\text { Systems, Man, and Cybernetics } \\
\text { (SMC) }\end{array}$ & $\begin{array}{c}\text { (Naranjo, Shojafar, } \\
\text { Abraham, et al., } \\
\text { 2016) } \\
\end{array}$ & YES \\
\hline & $\begin{array}{l}\text { Computers and Communication } \\
\text { (ISCC) }\end{array}$ & $\begin{array}{c}\text { (Brennand et al., } \\
\text { 2016) }\end{array}$ & $\mathrm{NO}$ \\
\hline & SOFTCOM Workshop & $(2016)$ & YES \\
\hline & $\begin{array}{l}\text { Cloud Computing Technology and Science } \\
\text { (CloudCom) }\end{array}$ & $\begin{array}{c}\text { (Hosseinpour et al., } \\
\text { 2016) }\end{array}$ & YES \\
\hline & Wireless Communications & $\begin{array}{c}\text { (Masip-Bruin, Marín- } \\
\text { Tordera, Gómez, } \\
\text { Barbosa, \& Alonso, } \\
\text { 2016) } \\
\end{array}$ & NO \\
\hline & Collaboration and Internet Computing (CIC) & $\begin{array}{l}\text { (Rauniyar, Engelstad, } \\
\text { \& Feng, 2016) }\end{array}$ & NO \\
\hline & $\begin{array}{l}\text { Globecom Workshops } \\
\text { (GC Wkshps) }\end{array}$ & $\begin{array}{c}\text { (Eriksson, Dan, \& } \\
\text { Fodor, 2016) }\end{array}$ & $\mathrm{NO}$ \\
\hline & Smart Cities Conference (ISC2) & $\begin{array}{c}\text { (Nazmudeen et al., } \\
\text { 2016) } \\
\end{array}$ & $\overline{\text { YES }}$ \\
\hline \multirow{2}{*}{ Springer } & "Signal, Networks, Computing, and Systems & $\begin{array}{c}\text { (Sahoo \& Sahoo, } \\
\text { 2017) }\end{array}$ & $\mathrm{NO}$ \\
\hline & $\begin{array}{c}\text { International Symposium on Intelligent and } \\
\text { Distributed Computing }\end{array}$ & (Pešić et al., 2017) & YES \\
\hline ACM & Cyber-Physical Systems & $\begin{array}{l}\text { (Vatanparvar, Chau, \& } \\
\text { Al Faruque, 2015) }\end{array}$ & YES \\
\hline
\end{tabular}




\begin{tabular}{|c|c|c|c|}
\hline \multirow{3}{*}{ Elsevier } & \begin{tabular}{|l} 
Journal of environmental \\
management
\end{tabular} & (Bratina et al., 2016) & $\mathrm{NO}$ \\
\hline & Journal of Pediatric Urology & $\begin{array}{c}\text { (Meenakshi- } \\
\text { Sundaram et al., } \\
\text { 2017) }\end{array}$ & NO \\
\hline & Future Generation Computer Systems & (Koo \& Hur, 2017) & $\mathrm{NO}$ \\
\hline \multirow{2}{*}{$\begin{array}{c}\text { Taylor \& Francis } \\
\text { Group }\end{array}$} & Cambridge Journal of Education & $\begin{array}{c}\text { (Liu, Hodgson, \& } \\
\text { Barry, 1998) }\end{array}$ & $\mathrm{NO}$ \\
\hline & Journal of Hospital Librarianship & (Heyd, 2012) & $\mathrm{NO}$ \\
\hline AMS & Numerical Weather Prediction & $\begin{array}{c}\text { (Zhou, Du, Ferrier, } \\
\text { McQueen, \& Dimego, } \\
\text { 2007) } \\
\end{array}$ & $\mathrm{NO}$ \\
\hline $\begin{array}{c}\text { University of Informat } \\
\text { ion } \\
\text { Science \& Technology }\end{array}$ & Environmental Science & (Xing-na, 2012) & $\mathrm{NO}$ \\
\hline $\begin{array}{l}\text { State of North } \\
\text { Carolina } \\
\end{array}$ & $\begin{array}{l}\text { Considerations for the Management of } \\
\text { Discharge of Fats, Oil and Grease }\end{array}$ & (Force, 2002) & $\mathrm{NO}$ \\
\hline MECS & International Journal of information & (Agarwal et al., 2016) & YES \\
\hline IET & Emerging Advances and Applications & $\begin{array}{c}\text { (Nikoloudakis et al., } \\
\text { 2017) } \\
\end{array}$ & $\mathrm{NO}$ \\
\hline $\begin{array}{c}\text { Hindawi } \\
\text { Publishing } \\
\text { Corporation }\end{array}$ & Mobile Information Systems & (Park \& Yoo, 2017) & YES \\
\hline
\end{tabular}

\section{Review of the selected papers}

Eleven selected articles are reviewed in this section according to the mentioned criteria. The advantages and disadvantages of the selected methods in energy, data, and resource management categories are discussed and explained as well.

\subsection{Energy management strategies}

Fog computing expends the paradigm of cloud computing to the edge of the network, that also may support IoT with the capability to pre-process the data in removing the low latency, which is useful in energy management systems [36]. Therefore, controlling power generation and consumption is possible by energy management. The first step in this section is to discuss the selected energy management strategies in the fog computing and describe their main features.

\subsubsection{Overview of selected articles}


An energy management strategy as a service on the fog computing was proposed by Vatanparvar et al. (2015). They stated that the flexibility, connectivity, data secrecy, and realtime features required for energy management are authorized by performing fog computing statement. The user has the ability to control the energy management policy by providing open source hardware/software. So, it decreases the implementation cost and time-to-market. The energy management strategy as a service requires two prototypes of home energy management and micro grid-level energy management for its presentation on the fog computing in different areas. However, this method suffers from low security and high complexity. [37]

Also, Naranjo, Shojafar, Abraham, et al. (2016) proposed a New Stable Election Protocol (NSEP) to manage and control the advanced nodes and select cluster heads in the heterogeneous fog-supported Wireless Sensor Networks (WSNs). Magnifying the lifetime of network and saving energy are considered as the main gains of the NSEP. The network lifetime has lesser energy consumption in this protocol compared to the traditional ones. But, the performance of high power sensors needs more studies in this paper. [38]

Finally, Nazmudeen et al. (2016) proposed a framework for distributed data aggregation by sending the data to the centralized fog storage space. To implement this method with acceptable efficiency and success, data collection and transmission infrastructures should be in the correct position. Smart meters are necessary to scale the energy utilization details and patterns. Data aggregation units are helpful in transferring to the Meter Data Management system (MDMS). The pure data accumulated from the consumers are very huge and they fall under the class of big data popularly. [39] All the collected data from smart meters are kept in a rationalize place for processing the energy request. This approach is becoming a bottleneck for efficient data collection due to limited bandwidth size of Power Line Communication (PLC). Using a fogbased approach for data aggregation could improve the response time according to the simulations, but this work should be more practical.

\subsubsection{Summary and comparison}

The energy management strategies discussed and reviewed in the previous subsection. Accordingly, the proposed methods in [40] suffers from low security in the fog environments. The response time is improved by using these strategies. Table 5 shows the advantages, disadvantages, and comparison of the reviewed energy management strategies. 
Reference

\section{Al Faruque and Vatanparvar (2016)}

Naranjo, Shojafar, Abraham, et al. (2016)
Main idea

Implementing the energy management with the customized control-as-services
Advantages

Low cost

Low time-to-market
Disadvantages

High complexity
Prolonging the stable period of fogsupported sensor networks by maintaining balanced energy consumption.
Low response time

High lifetime

Low performance

$\begin{array}{ccc}\text { Nazmudeen et al. } & \begin{array}{c}\text { Framework for distributing data } \\ \text { aggregation approach with the help } \\ \text { of fog computing architecture. }\end{array} & \text { High efficiency } \\ \end{array}$

\subsection{Data management strategies}

Data management is another important service level objective that are highly required to be achieved for the efficient performance of Fog computing. In different research works data management in Fog computing has been discussed from different perspectives [41]. As we know, relying on the current technologies such as grid and cloud computing is not an effective way to mention the necessities of the big data management. Hence, the reduction of complexity, the improvement of management and the promotion of big data require new technologies. Preprocessing of raw sensory data is an effective means for reducing the load of the big data in the computing environments. The raw and passive data generated by networks sensors are designed again to self-managed, which is considered as the benefits of fog computing. The intelligent data cells with reduced size are able to become more meaningful information. Therefore, fog computing refers to data management as one of the main issues. The selected data management strategies in the fog computing and their important attributes are discussed in this part.

\subsubsection{Overview of the selected articles}


Also, a communication system to consider the requirement to store the large data is proposed by Naranjo, Shojafar, Vaca-Cardenas, et al. (2016). [42] While critical time-sensitive data is stored and analyzed at the network edge, less time-sensitive data can go to the cloud for long Fog Data Services (FDS) is an IoT software product that runs in the network and aims to transform raw data from sensors and endpoints to actionable information. So, their operations can use data immediately and reduces the stress of the network. They have also provided an overview of existing works about the integration of fog computing in the future SG architectures which support Band Width (Meenakshi-Sundaram et al.). Using the BD SG applications on fog computing is one of the more valuable techniques to win the challenges related to traditional power grid management, despite the existence of some technical problems inherent fog computing. They also suggested a list of general requirements for BD SG applications on fog computing, being these necessities essential to design and implement effective and efficient BD applications. High security and efficiency are considered as the advantages of the proposed strategy, but high runtime and complexity are its weaknesses. [43]

Another solution for data management in the fog computing proposed by Dastjerdi and Buyya (2016). [44] The IoT sensors are capable to spread in different surrounding such as roads, medical centers, and farms due to the ideal performance in the system. When the system generates the information from the sensors, fog devices such as nearby gateways and private clouds manage data analytics. Therefore, it is necessary to check various resource management and scheduling techniques, including the placement, consolidation, and migration of stream processing operators, application modules, and tasks in order to enable real-time analytics in the fog computing. They have developed an iFogSim as an open source simulator. iFogSim provides the facilities for modeling and simulating the fog-computing environments for the evaluation of resource-management and scheduling policies across cloud and edge resources under many scenarios, based on their impact on latency, energy consumption, and operational costs. It makes low traffic and low cost, but it suffers from high latency and more decisionmaking times.

Finally, Park and Yoo (2017) described a method to take advantage of fog computing and Software Defined Networks (SDN) in the connected vehicle environment, where communication channels are unstable and the topology changes frequently. A controller knows the current state of the network by maintaining the most recent network topology. They proposed an approach to utilize mobility information to reduce control message overhead by adjusting the period of beacon messages and support efficient failure recovery. This mechanism 
has two main benefits such as low failure recovery time and low overhead but low security still remains as a problem. [45]

\subsubsection{Summary and comparison}

The previous subsection discussed and reviewed data management strategies. High latency and complexity for data management are considered as the disadvantages of these methods. The advantages, disadvantages, and comparison of the reviewed data management strategies are shown in Table 6.

Table 6. A side-by-side comparison of the reviewed data management mechanisms in the fog computing

\begin{tabular}{|c|c|c|c|}
\hline Reference & Main idea & Advantages & Disadvantages \\
\hline $\begin{array}{l}\text { Dastjerdi and Buyya } \\
\text { (2016) }\end{array}$ & $\begin{array}{l}\text { Designing e method to overcome } \\
\text { some limitations of fog computing } \\
\text { like large amounts of data. }\end{array}$ & $\begin{array}{l}\text { Low traffic } \\
\text { Low cost }\end{array}$ & $\begin{array}{l}\text { High latency } \\
\text { High decision- } \\
\text { making times }\end{array}$ \\
\hline $\begin{array}{l}\text { Naranjo, Shojafar, } \\
\text { Vaca-Cardenas, et al. } \\
(\mathbf{2 0 1 6 )}\end{array}$ & $\begin{array}{l}\text { Fog Nodes (FNs) can gather big data } \\
\text { and transport them to the remote } \\
\text { cloud, in order to perform in-depth } \\
\text { data analysis. }\end{array}$ & $\begin{array}{l}\text { High efficiency } \\
\text { High security }\end{array}$ & $\begin{array}{l}\text { High cost } \\
\text { High complexity }\end{array}$ \\
\hline Park and Yoo (2017) & $\begin{array}{l}\text { A method utilizing the network state } \\
\text { information to overcome unstable } \\
\text { communication in the fog computing } \\
\text { and SDN-based connected vehicle } \\
\text { environment. }\end{array}$ & $\begin{array}{l}\text { Low overhead } \\
\text { Low failure } \\
\text { recovery time }\end{array}$ & $\begin{array}{l}\text { High cost } \\
\text { Low security }\end{array}$ \\
\hline
\end{tabular}

\subsection{Resource management strategies}

The extension of network resources near the underlying networks is performed by fog computing. The generated data is analyzed and data filtering is performed through fog computing and also it generates more meaningful and essential data. Then, the fog resources store data temporarily. Once the data are uploaded to the cloud, local storage is not required. Then, that data is removed from the storage media [46, 57-60]. In a heterogeneous ambiance, resource management functions typically have various execution time and energy utilization characteristics when performed on different devices.

\subsubsection{Overview of the selected articles}


The extension of the IoT and their integration with cloud computing discussed by Aazam and Huh (2015) to provide more useful services to the user and efficient utilization of resources. Combination of cloud computing with IoTs, termed as Cloud of Things (CoT) provides ease of management for the growing media content and other data. Besides this, some attractive features such as resource provisioning, service discovery, service creation, and ubiquitous access can be offered by CoT. The proposed model covers the issues of resource prediction, advance reservation, and pricing for new and existing IoT customers based on fog computing. The experiment was done using Java, while the model was evaluated using CloudSim toolkit. However, this method suffers from low QoS and mobility to the obtained results.

$\mathrm{Gu}$ et al. (2015) have proposed a method in which fog computation and MCPS ${ }^{1}$ are incorporated to build supported MCPS. Specifically, task distribution, base station association and virtual machine placement are investigated towards cost output. They first formulated the problem as a Mixed-Integer Non-linear/Linear Program (MINLP) and then linearized it into a Mixed Integer Linear Programming (MILP). High solving computational complexity and costefficient resource management are considered as the benefits of this article. But, this method does not provide a solution to enhance security and traffic. [47]

Pešić et al. (2017) have proposed a solution, which performs decision-making for a smart actuation based on analysis of sensory data streams, and context informed fog computing resource. It mainly focuses either on smart actuation enabled through insightful data analysis and machine learning or on managing fog system itself in order to improve performance and efficiency. The solution shows cases how one software framework can be used to achieve both. Proof of concept experiments is executed on a fog computing testbed validate the performance of the solution in improving resilience and responsiveness of the fog computing system in the context of topology changes. But, since the presented solution is comprised of three main mechanisms, it makes the work a bit complex. [48]

Furthermore, Agarwal et al. (2016) have presented an effective architecture and algorithm for resources provisioning in the fog environment using virtualization method. The significance of efficient resource allocation and its related concepts are provided in this paper. Also, different types of current algorithms related to optimal resource allocation and different scheduling

\footnotetext{
${ }^{1}$ Montgomery County Public Schools
} 
techniques are discussed in this method. This idea helps to minimize response time and cost, however, this approach suffers from low availability and complexity. [49]

Finally, Cardellini et al. (2015) have presented a general formulation of the optimal assignment problem for data stream processing applications in which the heterogeneity of computing and networking resources are considered. Different QoS requirements of the applications are optimized and they have considered the end-to-end latency, availability and exchanged datarate among operators. So, these metrics are the advantages of using this formulation, but it suffers from profit maximization. A complete experimental evaluation of the proposed solution is provided using two sets of data stream processing applications. The first one is characterized by a simple topology with various requirements and the other one contains some well-known applications. The distributed QoS-aware scheduler outperforms the centralized default one, improves the application performance and raises the system with runtime adaptation abilities. So, complex topologies with many operators may cause instability to decrease the availability of data stream processing application. [50]

\subsubsection{Summary and comparison}

The selected resource management strategies in the fog computing were discussed in the previous section. Availability is considered as an important factor in most of the resource management. Table 7 shows a side-by-side comparison, advantages, and disadvantages of the discussed methods.

Table 7. A side-by-side comparison of the resource management in fog computing

\begin{tabular}{|c|c|c|c|}
\hline Reference & Main idea & Advantages & Disadvantages \\
\hline \multirow{3}{*}{$\begin{array}{l}\text { Aazam and Huh } \\
\text { (2015) }\end{array}$} & \multirow{3}{*}{$\begin{array}{l}\text { A resource management framework } \\
\text { for manage resources, perform data } \\
\text { filtration, preprocessing, and security } \\
\text { measures }\end{array}$} & Low latency & \\
\hline & & Low delay & Low security \\
\hline & & High validity & Low scalability \\
\hline \multirow{2}{*}{ Gu et al. (2015) } & \multirow{2}{*}{$\begin{array}{l}\text { Motivating to integrate fog } \\
\text { computation and MCPS to build fog } \\
\text { computing supported MCPS }\end{array}$} & Low cost & Low security \\
\hline & & Low complexity & High traffic \\
\hline
\end{tabular}


Decision-making for a smart actuation based on analysis of sensory data Pešić et al. streams, and context informed fog (2017) computing resource and service provisioning management based on Flexibility topology changes

Response time

Complexity

Low cost High complexity

Agarwal et al.

Efficient resource allocation algorithm

(2016) to proper utilization of the resources and to reduce the congestion by using the intermediate fog layer

High efficiency

Low availability

Cardellini et al. (2015)
A distributed and self-adaptive QoSaware scheduler for storm, an open source data stream processing system.
High efficiency

High complexity

High run-time

Low availability

\section{Discussions and Results}

The most general fog management strategies were described in the previous sections. They include energy management, data management, and resource management. The energy management strategies can improve the efficiency by reducing the power consumption. It is suitable for energy managing in fog environment because it helps to preserve more energy. The data management strategies are also suitable for fog environment because it has low latency for data. Also, this management strategy has ubiquitous access compared to other strategies. The resource management strategies are suitable to manage fog environment and focus on optimal solution and flexibility. Moreover, data-based and energy-based strategies have high cost but the resource-based strategies have low cost. Furthermore, high flexibility of the resource management is necessary to manage the fog service, because it can be used for achieving an optimal solution by using most of the offered algorithms in articles.

Table 4 shows the selected articles and their QoS parameters. The focus of researchers on QoS parameters is shown in Fig. 5 . The cost is $18 \%$, time is $24 \%$, complexity is $16 \%$, efficiency is $14 \%$, security is $10 \%$, availability is $6 \%$ and other parameters are about $12 \%$. The cost and time attracted more attention according to the results. There should be more focus on security in the future. Also, reducing cost and improving efficiency can be considered as the main goals of fog management in the future. 


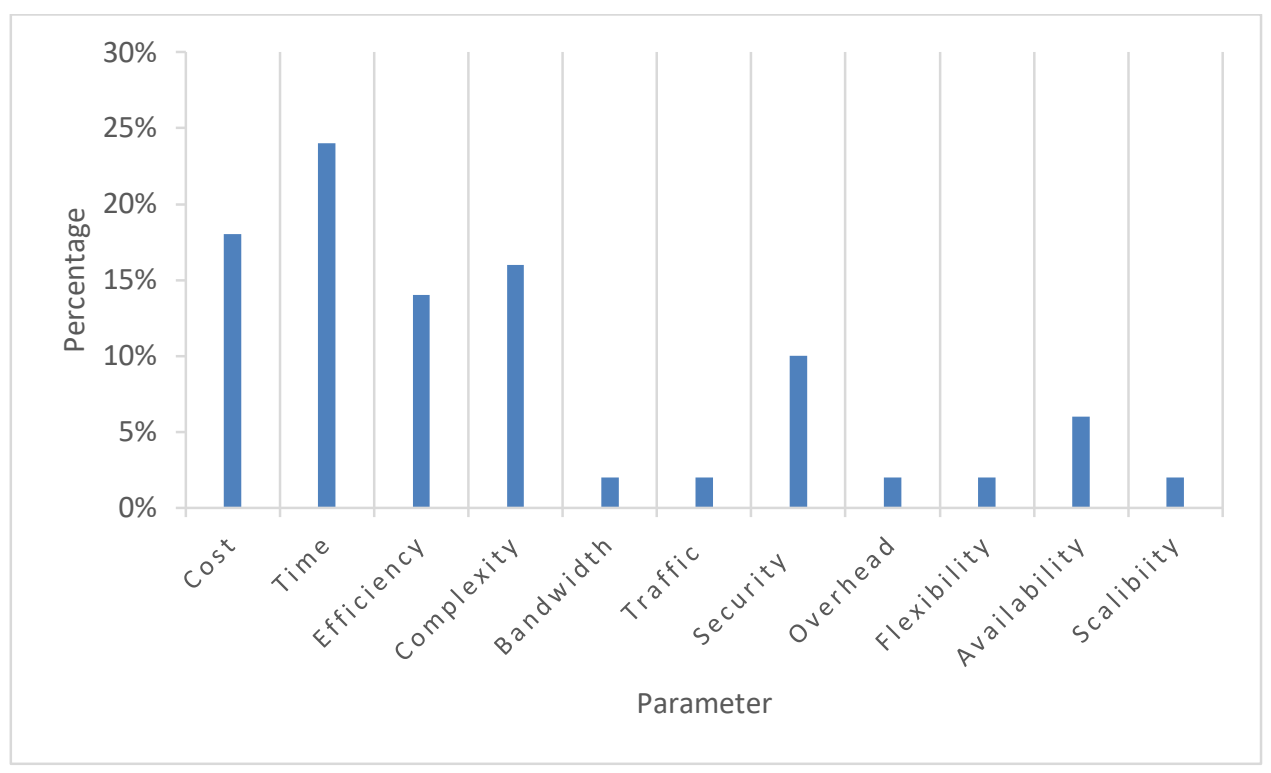

Fig 5. Considered parameters in the selected articles

\section{Open Issues}

This section presents the research directions in development and improvement of management strategies in the fog computing. These researches have not been studied comprehensively and completely up to now. Also, the management strategies have not a single mechanism to address all QoS parameters in fog. From this review, there are still a lot of works to be done in the field of management in fog computing. Therefore, some open important research problems are discussed in this section in three categories, including energy, data and resource management. [51]

The simulator-based tools are applied in most of the research for evaluations. Therefore, one of the future challenges is to perform the discussed approaches in the real world. Also, in the reviewed papers, some factors such as scalability, and time are considered while other parameters such as cost, efficiency and etc. are ignored. Therefore, considering the missed parameters is very interesting. Furthermore, analyzing the user's satisfaction is very interesting for future management strategies. In addition, considering more optimized and flexible solutions is very important in developing a personalization framework. The future research has more focus on designing and performance of a prototyping platform in fog computing. The full-fledged fog platform implementations and the performance of optimizations in data management are considered as the future challenges. [52, 61-64] 
Moreover, mobility factor will be incorporated and added in the future to extend these works regarding more diverse services and QoS and considering fog computing concept. It is also necessary to establish a QoS-aware scheduling over fog infrastructures to overcome the highlighted weaknesses. Also, these papers may be extended towards other issues and matrices in such as task migration and load balancing by which the efficiency and performance of the system can be improved in fog environment. [53 - 60]

Finally, to be sure, not every kind of computing application is, nor will need to be thought of as, a "fog computing" opportunity, but a lot of the most interesting ones in business environments are going to be. We're starting to witness a massive shift away from the centralized computing model that enabled the cloud and toward the distribution of more computing power back out to the edge of the network. The reason for this is that big-picture applications like autonomous driving, smart cities, smart agriculture, smart homes and even remote medicine all have requirements that can't always be addressed by the cloud in its current form. We're seeing a massive shift away from the centralized computing model that enabled the cloud and toward the distribution of more computing power back out to the edge of the network. Even with all these interesting efforts, we aren't going to see a traditional cloud, network, data center or common endpoints going away. These new fog computing efforts are typically created in addition to these still-critical infrastructure elements, sometimes through the use of small fog nodes. In fact, several companies have started building proprietary solutions that leverage some of these ideas to create unique opportunities for themselves. As with most web-based initiatives, however, it's going to take more open efforts - like those being driven by the Open Fog Consortium to gain more widespread success. [54-68]

\section{Conclusion}

This study presents a systematic review of management strategies in the fog computing. Different fog management systems and many open issues are discussed through an in-depth analysis of 11 primary studies with basic 75 papers from our search query. Our research proves management as an ascending mechanism through answering three research questions and a new paradigm is ensured by increasing fog performance and the resource utilization. Each input request is distributed efficiently and fairly. The articles are classified into three sub-domains based on energy management, data management, and resource management studies. Also, the advantages and disadvantages of many fog management algorithms are presented. Many impressive management strategies are developed due to the challenges in this algorithm. Proper 
management has the ability to keep minimum resource expanding which will finish further reduction of energy consumption. In general, fog management strategies in computing environment still need improvements in the heterogeneity of its environment in order to become an on-demand technique, decrease the associated overhead and improve the performance. Exclusively, the management primary purposes, current challenges, open issues, approaches and mechanisms in fog systems are summarized through answering the questions. In the future, the researchers will develop the maturity and adoption of management strategies in fog computing through the results of this study.

\section{References}

2. Aazam, M., \& Huh, E.-N. (2015). Fog computing micro datacenter based dynamic resource estimation and pricing model for IoT. Paper presented at the Advanced Information Networking and Applications (AINA), 2015 IEEE 29th International Conference on.

3. Afrooz, S., \& Navimipour, N. J. (2018). Memory Designing Using Quantum-Dot Cellular Automata: Systematic Literature Review, Classification and Current Trends. Journal of Circuits, Systems and Computers, 1730004.

4. Agarwal, S., Yadav, S., \& Yadav, A. K. (2016). An Efficient Architecture and Algorithm for Resource Provisioning in Fog Computing. International Journal of Information Engineering and Electronic Business, 8(1), 48.

5. Al Faruque, M. A., \& Vatanparvar, K. (2016). Energy management-as-a-service over fog computing platform. IEEE Internet of Things Journal, 3(2), 161-169.

6. Alam, M., \& Shakil, K. A. (2015). Recent developments in cloud based systems: state of art. arXiv preprint arXiv:1501.01323.

7. Amendola, D., Cordeschi, N., \& Baccarelli, E. (2016). Bandwidth Management VMs Live Migration in Wireless Fog Computing for 5G Networks. Paper presented at the Cloud Networking (Cloudnet), 2016 5th IEEE International Conference on.

8. Bratina, B., Šorgo, A., Kramberger, J., Ajdnik, U., Zemljič, L. F., Ekart, J., \& Šafarič, R. (2016). From municipal/industrial wastewater sludge and FOG to fertilizer: A proposal for economic sustainable sludge management. Journal of environmental management, 183, 1009-1025.

9. Brennand, C. A., da Cunha, F. D., Maia, G., Cerqueira, E., Loureiro, A. A., \& Villas, L. A. (2016). FOX: A traffic management system of computer-based vehicles FOG. Paper presented at the Computers and Communication (ISCC), 2016 IEEE Symposium on.

10. Buyya, R., \& Dastjerdi, A. V. (2016). Internet of Things: Principles and paradigms: Elsevier.

11. Cardellini, V., Grassi, V., Lo Presti, F., \& Nardelli, M. (2016). Optimal operator placement for distributed stream processing applications. Paper presented at the Proceedings of the 10th ACM International Conference on Distributed and Event-based Systems.

12. Cardellini, V., Grassi, V., Presti, F. L., \& Nardelli, M. (2015). On QoS-aware scheduling of data stream applications over fog computing infrastructures. Paper presented at the Computers and Communication (ISCC), 2015 IEEE Symposium on. 
13. Charband, Y., \& Navimipour, J. N. (2018). Knowledge sharing mechanisms in the education: a systematic review of the state of the art literature and recommendations for future research. Kybernetes.

14. Chiregi, M., \& Jafari Navimipour, N. (2018). Cloud computing and trust evaluation: A systematic literature review of the state-of-the-art mechanisms. Journal of Electrical Systems and Information Technology. doi:https://doi.org/10.1016/j.jesit.2017.09.001

15. Cirani, S., Ferrari, G., Iotti, N., \& Picone, M. (2015). The iot hub: a fog node for seamless management of heterogeneous connected smart objects. Paper presented at the Sensing, Communication, and Networking-Workshops (SECON Workshops), 2015 12th Annual IEEE International Conference on.

16. Dastjerdi, A. V., \& Buyya, R. (2016). Fog Computing: Helping the Internet of Things Realize Its Potential. Computer, 49(8), 112-116.

17. Dastjerdi, A. V., Gupta, H., Calheiros, R. N., Ghosh, S. K., \& Buyya, R. (2016). Fog computing: Principles, architectures, and applications. arXiv preprint arXiv:1601.02752.

18. Dsouza, C., Ahn, G.-J., \& Taguinod, M. (2014). Policy-driven security management for fog computing: Preliminary framework and a case study. Paper presented at the Information Reuse and Integration (IRI), 2014 IEEE 15th International Conference on.

19. Eriksson, E., Dan, G., \& Fodor, V. (2016). Radio and Computational Resource Management for Fog Computing Enabled Wireless Camera Networks. Paper presented at the Globecom Workshops (GC Wkshps), 2016 IEEE.

20. Force, F. T. (2002). Considerations for the Management of Discharge of Fats, Oil and Grease (FOG) to Sanitary Sewer Systems: State of North Carolina.

21. Gu, L., Zeng, D., Guo, S., Barnawi, A., \& Xiang, Y. (2015). Cost-efficient resource management in fog computing supported medical cps. IEEE Transactions on Emerging Topics in Computing.

22. Gupta, H., Vahid Dastjerdi, A., Ghosh, S. K., \& Buyya, R. (2017). iFogSim: A toolkit for modeling and simulation of resource management techniques in the Internet of Things, Edge and Fog computing environments. Software: Practice and Experience, 47(9), 1275-1296.

23. Hazratzadeh, S., Hazratzadeh, S., Jafari Navimipour, N., \& Jafari Navimipour, N. (2016). Colleague recommender system in the Expert Cloud using features matrix. Kybernetes, 45(9), 1342-1357.

24. Heyd, M. (2012). Getting Started With Cloud Computing: A LITA Guide edited by Edward M. Corrado and Heather Lee Moulaison: (2011). New York, NY: NealSchuman Publishers, 214 pages, \$65.00, softcover, ISBN: 978-1-55570-749-1: Taylor \& Francis.

25. Hosseinpour, F., Plosila, J., \& Tenhunen, H. (2016). An Approach for Smart Management of Big Data in the Fog Computing Context. Paper presented at the Cloud Computing Technology and Science (CloudCom), 2016 IEEE International Conference on.

26. Hu, P., Dhelim, S., Ning, H., \& Qiu, T. (2017). Survey on fog computing: architecture, key technologies, applications and open issues. Journal of Network and Computer Applications.

27. Jennings, B., \& Stadler, R. (2015). Resource management in clouds: Survey and research challenges. Journal of Network and Systems Management, 23(3), 567-619.

28. Koo, D., \& Hur, J. (2017). Privacy-preserving deduplication of encrypted data with dynamic ownership management in fog computing. Future Generation Computer Systems. 
29. Liu, F., Hodgson, J., \& Barry, T. (1998). Effects of grazing sequence and condensed tannins on ingestive behaviour, herbage intake, and performance of lambs grazing Yorkshire fog pasture. New Zealand Journal of Agricultural Research, 41(3), 359-366.

30. Luan, T. H., Gao, L., Li, Z., Xiang, Y., Wei, G., \& Sun, L. (2015). Fog computing: Focusing on mobile users at the edge. arXiv preprint arXiv:1502.01815.

31. M Shojafar, N. C. (2016). Energy-efficient adaptive resource management for real-time vehicular cloud services

32. Mahmud, R., Kotagiri, R., \& Buyya, R. (2018). Fog computing: A taxonomy, survey and future directions Internet of Everything (pp. 103-130): Springer.

33. Manvi, S. S., \& Shyam, G. K. (2014). Resource management for Infrastructure as a Service (IaaS) in cloud computing: A survey. Journal of Network and Computer Applications, 41, 424-440.

34. Masip-Bruin, X., Marín-Tordera, E., Gómez, A., Barbosa, V., \& Alonso, A. (2016). Will it be cloud or will it be fog? F2C, A novel flagship computing paradigm for highly demanding services. Paper presented at the Future Technologies Conference (FTC).

35. Meenakshi-Sundaram, B., Furr, J., Malm-Buatsi, E., Boklage, B., Nguyen, E., Frimberger, D., \& Palmer, B. (2017). Reduction in surgical fog with a warm humidified gas management protocol significantly shortens procedure time in pediatric robotassisted laparoscopic procedures. Journal of Pediatric Urology.

36. Mohammadi, S. Z., \& Navimipour, N. J. (2017). Invalid cloud providers' identification using the support vector machine. INTERNATIONAL JOURNAL OF NEXTGENERATION COMPUTING, 8(1).

37. Naranjo, P. G. V., Shojafar, M., Abraham, A., \& Baccarelli, E. (2016). A new stable election-based routing algorithm to preserve aliveness and energy in fog-supported wireless sensor networks. Paper presented at the Systems, Man, and Cybernetics (SMC), 2016 IEEE International Conference on.

38. Naranjo, P. G. V., Shojafar, M., Vaca-Cardenas, L., Canali, C., Lancellotti, R., \& Baccarelli, E. (2016). Big Data Over SmartGrid-A Fog Computing Perspective. Paper presented at the SOFTCOM Workshop.

39. Nazmudeen, M. S. H., Wan, A. T., \& Buhari, S. M. (2016). Improved throughput for power line communication (plc) for smart meters using fog computing based data aggregation approach. Paper presented at the Smart Cities Conference (ISC2), 2016 IEEE International.

40. Nikoloudakis, Y., Panagiotakis, S., Markakis, E., Mastorakis, G., Mavromoustakis, C., $\&$ Pallis, E. (2017). Towards a FOG-enabled navigation system with advanced crosslayer management features and IoT equipment. Cloud and Fog Computing in 5G Mobile Networks, 171.

41. O Skarlat, M. N., S Schulte. (2017). Towards QoS-aware fog service placement.

42. Panciatici, P., Bareux, G., \& Wehenkel, L. (2012). Operating in the fog: Security management under uncertainty. IEEE Power and Energy Magazine, 10(5), 40-49.

43. Park, S., \& Yoo, Y. (2017). Network Intelligence Based on Network State Information for Connected Vehicles Utilizing Fog Computing. Mobile Information Systems, 2017.

44. Pešić, S., Tošić, M., Iković, O., Ivanović, M., Radovanović, M., \& Bošković, D. (2017). Context Aware Resource and Service Provisioning Management in Fog Computing Systems. Paper presented at the International Symposium on Intelligent and Distributed Computing.

45. Popentiu-Vladicescu, F., \& Albeanu, G. (2017). Software reliability in the fog computing. Paper presented at the Innovations in Electrical Engineering and Computational Technologies (ICIEECT), 2017 International Conference on. 
46. Rauniyar, A., Engelstad, P., \& Feng, B. (2016). Crowdsourcing-Based Disaster Management Using Fog Computing in Internet of Things Paradigm. Paper presented at the Collaboration and Internet Computing (CIC), 2016 IEEE 2nd International Conference on.

47. Saharan, K., \& Kumar, A. (2015). Fog in comparison to cloud: A survey. International Journal of Computer Applications, 122(3).

48. Sahoo, K. S., \& Sahoo, B. (2017). Sdn architecture on fog devices for realtime traffic management: A case study. Paper presented at the Proceedings of the International Conference on Signal, Networks, Computing, and Systems.

49. Santos, J., Wauters, T., Volckaert, B., \& De Turck, F. (2017). Fog Computing: Enabling the Management and Orchestration of Smart City Applications in 5G Networks. Entropy, 20(1), 4.

50. Sheikholeslami, F., \& Navimipour, N. J. (2017). Service allocation in the cloud environments using multi-objective particle swarm optimization algorithm based on crowding distance. Swarm and Evolutionary Computation, 35(Supplement C), 53-64. doi:https://doi.org/10.1016/j.swevo.2017.02.007

51. Shi, J., Wan, J., Yan, H., \& Suo, H. (2011). A survey of cyber-physical systems. Paper presented at the Wireless Communications and Signal Processing (WCSP), 2011 International Conference on.

52. Shi, W., Cao, J., Zhang, Q., Li, Y., \& Xu, L. (2016). Edge computing: Vision and challenges. IEEE Internet of Things Journal, 3(5), 637-646.

53. Souri, A., Navimipour, N. J., \& Rahmani, A. M. (2018). Formal verification approaches and standards in the cloud computing: A comprehensive and systematic review. Computer Standards \& Interfaces. doi:https://doi.org/10.1016/j.csi.2017.11.007

54. Stojmenovic, I., Wen, S., Huang, X., \& Luan, H. (2016). An overview of Fog computing and its security issues. Concurrency and Computation: Practice and Experience, 28(10), 2991-3005. doi:10.1002/cpe.3485

55. Tomovic, S., Yoshigoe, K., Maljevic, I., \& Radusinovic, I. (2017). Software-Defined Fog Network Architecture for IoT. Wireless Personal Communications, 92(1), 181196. doi:10.1007/s11277-016-3845-0

56. Vakili, A., \& Navimipour, N. J. (2017). Comprehensive and systematic review of the service composition mechanisms in the cloud environments. Journal of Network and Computer Applications, 81, 24-36.

57. Vatanparvar, K., Chau, Q., \& Al Faruque, M. A. (2015). Home energy management as a service over networking platforms. Paper presented at the Innovative Smart Grid Technologies Conference (ISGT), 2015 IEEE Power \& Energy Society.

58. Xing-na, Y. (2012). 5, LI Xin-mei2, DENG Zen-grandeng3, DE Qing-yangzong4, YUAN Shuai5 (1. Key Laboratory of Meteorological Disaster of Ministry of Education, Nanjing University of Information Science \& Technology, Nanjing 210044, China; 2. Chinese Civil Aviation Hulunbuir Air Traffic Management Station, Hulunbuir 021008, China; 3. Meteorological Bureau of Lazi County, Xigaze City, The Tibet Autonomous Region, Lazi 858100, China; 4. Meteorological Bureau of Chayu County, Linzhi City, The Tibet Autonomous Region, Chayu 860600, China; 5. School of Atmospheric Physics, Nanjing Univwrsity of Information Science \& Technology, Nanjing 210044, China); Optical Properties of Aerosol During Haze-Fog Episodes in Beijing [J]. Environmental Science, 4.

59. Yang, T., Cui, Z., Wang, R., Zhao, J., Su, Z., \& Deng, R. (2017). A multi-vessels cooperation scheduling for networked maritime fog-ran architecture leveraging SDN. Peer-to-Peer Networking and Applications. doi:10.1007/s12083-017-0569-1 
60. Yao, H., Bai, C., Xiong, M., Zeng, D., \& Fu, Z. (2017a). Heterogeneous cloudlet deployment and user-cloudlet association toward cost effective fog computing. Concurrency and Computation: Practice and Experience, 29(16), e3975-n/a. doi:10.1002/cpe.3975

61. Yao, H., Bai, C., Xiong, M., Zeng, D., \& Fu, Z. (2017b). Heterogeneous cloudlet deployment and user-cloudlet association toward cost effective fog computing. Concurrency and Computation: Practice and Experience, 29(16).

62. Yaseen, Q., Albalas, F., Jararwah, Y., \& Al-Ayyoub, M. (2017). Leveraging fog computing and software defined systems for selective forwarding attacks detection in mobile wireless sensor networks. Transactions on Emerging Telecommunications Technologies.

63. Yi, S., Hao, Z., Qin, Z., \& Li, Q. (2015). Fog computing: Platform and applications. Paper presented at the Hot Topics in Web Systems and Technologies (HotWeb), 2015 Third IEEE Workshop on.

64. Yi, S., Li, C., \& Li, Q. (2015). A survey of fog computing: concepts, applications and issues. Paper presented at the Proceedings of the 2015 Workshop on Mobile Big Data.

65. Yi, S., Qin, Z., \& Li, Q. (2015). Security and privacy issues of fog computing: A survey. Paper presented at the International Conference on Wireless Algorithms, Systems, and Applications.

66. Zareie, B., \& Navimipour, N. J. (2016). The impact of electronic environmental knowledge on the environmental behaviors of people. Computers in Human Behavior, 59, 1-8.

67. Zhang, J., Li, Q., Wang, X., Feng, B., \& Guo, D. (2017). Towards fast and lightweight spam account detection in mobile social networks through fog computing. Peer-to-Peer Networking and Applications. doi:10.1007/s12083-017-0559-3

68. Zhou, B., Du, J., Ferrier, B., McQueen, J., \& Dimego, G. (2007). Numerical forecast of fog-central solutions. Paper presented at the 18th Conference on Numerical Weather Prediction, AMS. 